\title{
Botanical Briefs: Phytophotodermatitis Caused by Giant Hogweed (Heracleum mantegazzianum)
}

\author{
Kelly E. Flanagan, MS; Kaitlin Blankenship, MD; Laura Houk, MD
}

\section{PRACTICE POINTS}

- The public should be educated, especially during summer months, about how to identify giant hogweed, reduce exposure to the plant's phototoxin, and thus reduce the risk for severe phytophotodermatitis.

- Phytophotodermatitis should be included in the differential diagnosis when a patient presents with acute erythema and bullae in sun-exposed areas.

- Phytophotodermatitis can be treated by promptly washing the skin with soap and water, protecting the skin from exposure to UV light, and utilizing topical and oral steroids.

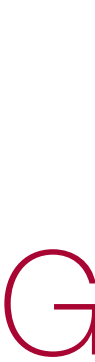

\author{
.
}

that typically reaches a height of 13 feet, with thick stems; large green leaves; and umbrella-shaped, flat-topped, radial clusters (umbels) of small individual white flowers ${ }^{1}$ (Figure 1). Because of the size and beauty of giant hogweed, it was widely planted in 19th century ornamental gardens in the United Kingdom and has since naturalized and spread throughout central Europe, Canada, and the United States. ${ }^{1,2}$ The plant most commonly is found in shady areas near rivers and woodlands. ${ }^{1}$

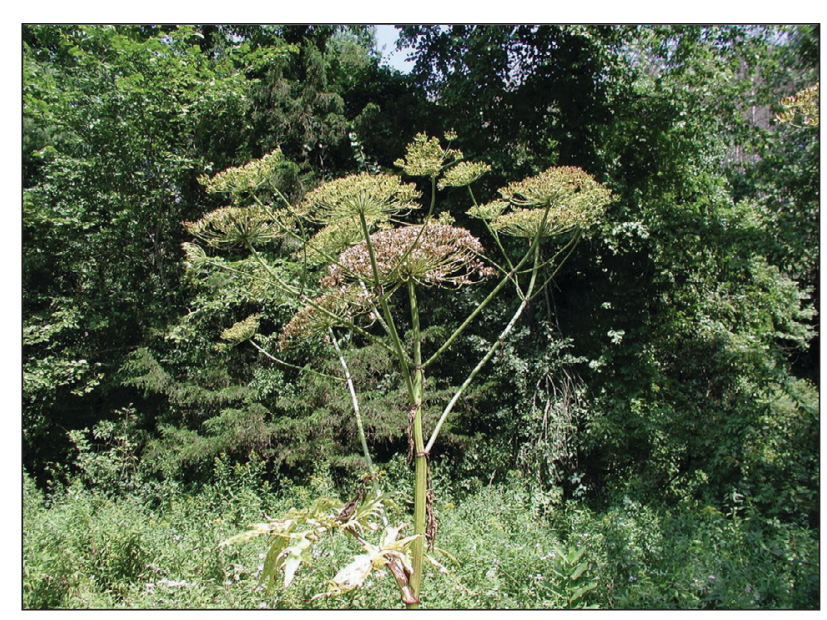

Giant hogweed (Heracleum mantegazzianum) is an invasive flowering weed that can reach a height of 13 feet. Although it is the appearance of the plant that can draw attention, contact with the sap in combination with exposure to the sun can result in severe phytophotodermatitis. Due to the growing prevalence of giant hogweed, the number of cases of $H$ mantegazzianum-associated phytophotodermatitis in the United States is increasing. We report the case of a 27 -year-old man who presented with a blistering rash on the neck and arms that developed 18 to 24 hours after trimming giant hogweed plants without photoprotection. A diagnosis of giant hogweed phytophotodermatitis should be considered for patients presenting with acute erythema and bullae in sun-exposed areas after exposure to the plant. Additionally, the public would benefit from education, especially during summer months, on identifying the plant and reducing phototoxin exposure to reduce the risk for phytophotodermatitis.

Cutis. 2021;108:251-253.

FIGURE 1. Giant hogweed (Heracleum mantegazzianum). ( ) Leslie J. Mehrhoff, University of Connecticut, Bugwood.org. Reproduced with permission.

Ms. Flanagan is from the University of Massachusetts Chan Medical School, Worcester. Drs. Blankenship and Houk are from the Department of Dermatology, University of Massachusetts, Worcester.

The authors report no conflict of interest.

Correspondence: Kaitlin Blankenship, MD, University of Massachusetts Memorial Healthcare, Hahnemann Campus, 281 Lincoln St, Worcester, MA 01605 (KaitlinChiara@yahoo.com).

doi:10.12788/cutis.0389 
Due to the invasive nature of the giant hogweed, its prevalence continues to grow, its eradication remains difficult, and reports of phytophotodermatitis are increasing in number and distribution. In fact, there has been widespread media coverage of the dangers of giant hogweed in the United Kingdom since $2016^{1}$ and in the United States in 2018 and 2019..$^{3-6}$

\section{Transmission}

Phytophotodermatitis is a type of nonimmunologic dermatitis caused by UV light reacting with a plant-based photosensitizing agent. In the case of giant hogweed, sap from the plant's fruits, leaves, and stem contain furocoumarins or psoralens. ${ }^{7}$ Upon activation by UVA radiation, furan rings of these compounds create reactive oxygen species and intercalate with DNA pyrimidine bases, which results in cellular death, damage to successive skin layers, and reduced wound healing at the cellular level. ${ }^{8}$ This effect is intensified with increased percutaneous absorption of furocoumarin, which can result from high temperature, humidity, skin infection, lack of protective clothing, and moist conditions. ${ }^{9}$

The highest concentration of phototoxic compounds is found in giant hogweed from June through August, ${ }^{7}$ which, in combination with people increasing their outdoor activity in the summer, results in a greater prevalence and severity of $H$ mantegazzianum phytophotodermatitis during summer months.

\section{Presentation}

Phytophotodermatitis caused by giant hogweed can range from burning and erythema to full-thickness chemical burns that require surgical debridement and skin grafting. ${ }^{8}$ After exposure to the offending agent, a harmful skin reaction can start within 15 minutes. After a latent period of approximately 24 hours, erythema, edema, and bullae can appear and generally peak by 72 hours. ${ }^{10}$ In addition to cutaneous injury, inhalation of giant hogweed traces can result in obstructive pulmonary symptoms. Eye contact can result in blindness. ${ }^{9}$

In addition to the rash caused by giant hogweed, a "weed-wacker dermatitis" or "strimmer rash" can be caused by the similar-appearing but smaller common hogweed (Heracleum sphondylium). Common hogweed is highly prevalent in the United States and often is confused with the larger giant hogweed because of tall stems and white, flat-topped flowers.

\section{Management}

Following contact with giant hogweed, a person should immediately avoid UV exposure and rinse the area with soap and water. UV radiation must be avoided for at least 48 hours. If erythema occurs, a topical steroid can be applied to the affected area; pain can be alleviated by a nonsteroidal anti-inflammatory drug. ${ }^{9}$

Further treatment might be required if bullous lesions are present. Small blisters can be punctured and drained; however, large blisters, extensive epidermal-dermal separation, and large areas of detached epidermis should simply be cleansed and dressed. An oral steroid also can be used to reduce inflammation in moderate and severe cases. Full-thickness injury might require surgical intervention. ${ }^{8}$

\section{Clinical Case}

A 27-year-old male landscaper presented to the emergency department with an increasingly painful blistering rash on the arms and neck of 1 day's duration. He noticed bright red skin and blisters 18 to 24 hours after trimming what he identified as shoulder-high giant hogweed plants.

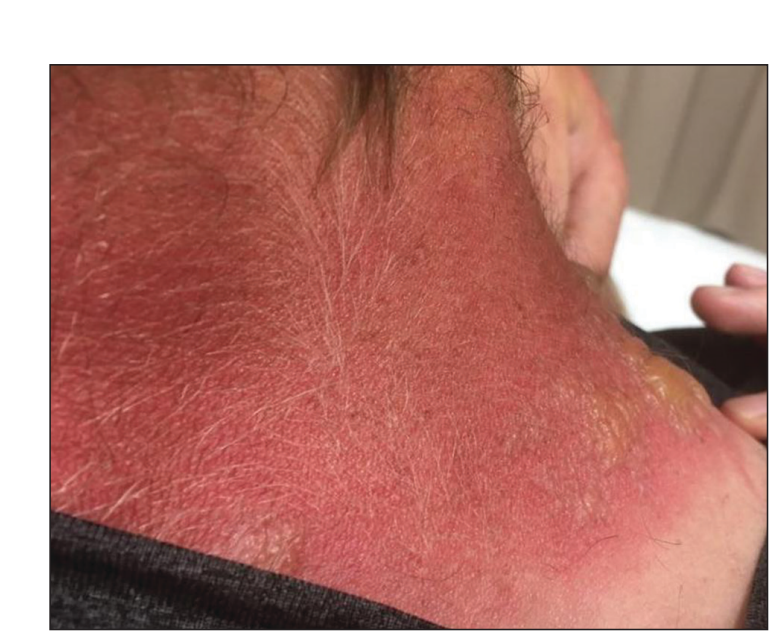

FIGURE 2. Bullae and erythema on the sun-exposed region of the posterior neck due to giant hogweed phytophotodermatitis.

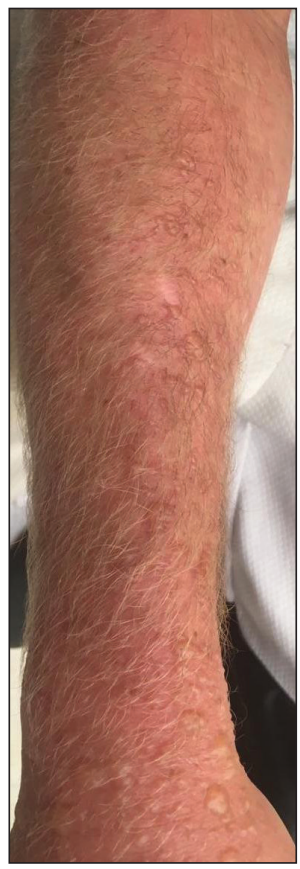

FIGURE 3. Vesicles and bullae on the sun-exposed region of the right arm due to giant hogweed phytophotodermatitis. 
Neither he nor his coworkers were wearing sunscreen or protective clothing as they cleared the plants for several hours. His coworkers developed similar rashes, but his rash was the most severe, requiring treatment in the emergency department.

Physical examination showed innumerable 2- to $10-\mathrm{mm}$, tense vesicles and bullae on a background of blanching erythema in a striking photodistribution along the neck (Figure 2) and arms (Figure 3). He had notable edema of both arms and several large 3- to 4 - $\mathrm{cm}$ bullae on the ventral aspects of the forearms.

The patient was diagnosed with severe phytophotodermatitis secondary to contact with $H$ mantegazzianum and was started on oral prednisone $70 \mathrm{mg}$ daily $(1 \mathrm{mg} / \mathrm{kg} / \mathrm{d})$, which was decreased by $10 \mathrm{mg}$ every 3 days until the course of treatment was complete. He also was instructed to apply mupirocin ointment to open areas and petroleum jelly to intact skin. Additionally, he was advised to practice strict photoprotection for the near and distant future.

Within several days after treatment began, the phytophotodermatitis dramatically improved, with complete resolution in 1 week. Postinflammatory hyperpigmentation resolved after several weeks.

\section{REFERENCES}

1. Baker B, Bedford J, Kanitkar S. Keeping pace with the media; giant hogweed burns-a case series and comprehensive review [published online December 26, 2016]. Burns. 2017;13:933-938. doi:10.1016/j.burns.2016.10.018

2. Klimaszyk P, Klimaszyk D, Piotrowiak M, et al. Unusual complications after occupational exposure to giant hogweed (Heracleum mantegazzianum): a case report. Int J Occup Med Environ Health. 2014;27:141-144. doi:10.2478/s13382-014-0238-Z

3. Zaveria M, Hauser C. Giant hogweed: a plant that can burn and blind you. but don't panic. New York Times. July 2, 2018. Accessed October 18, 2021. https://www.nytimes.com/2018/07/02/us/giant -hogweed-nyt.html

4. Hignett K. Giant hogweed: New York officials warn residents about dangerous plant that causes serious burns, blisters and scars. Newsweek. June25,2019.AccessedOctober18,2021.https://www.newsweek.com/giant -hogweed-new-york-dangerous-plant-burns-skin-sunlight-1445785

5. Eastman J. Toxic giant hogweed sap that burns, blisters skin found in Clark County. The Oregonian. Updated July 16, 2019. Accessed October 18, 2021. https://www.oregonlive.com/news/2019/07 /toxic-giant-hogweed-plant-that-burns-blisters-skin-found-in -clark-county.html

6. O'Kane C. Giant hogweed, plant that causes blindness and thirddegree burns, discovered in Virginia. CBS News. June 18, 2018. Accessed October 18, 2021. https://www.cbsnews.com/news /giant-hogweed-plant-causes-blindness-third-degree-burns -discovered-in-virginia-otherstates/

7. Pira E, Romano C, Sulotto F, et al. Heracleum mantegazzianum growth phases and furocoumarin content. Contact Dermatitis. 1989;21:300-303. doi:10.1111/j.1600-0536.1989.tb04747.x

8. Chan JCY, Sullivan PJ, O'Sullivan MJ, et al. Full thickness burn caused by exposure to giant hogweed: delayed presentation, histological features and surgical management. J Plast Reconstr Aesthet Surg. 2011;64:128-130. doi:10.1016/j.bjps.2010.03.030

9. Pfurtscheller K, Trop M. Phototoxic plant burns: report of a case and review of topical wound treatment in children. Pediatr Dermatol. 2014;31:E156-E159. doi:10.1111/pde.12396

10. Kavli G, Volden G: Phytophotodermatitis. Photodermatol. 1984;1:65-75. 